\title{
In-Situ Monitoring of the Curing of Epoxy Resins by Raman Spectroscopy
}

\author{
L. Merad ${ }^{1,3,4}$, M. Cochez ${ }^{1}$, S. Margueron ${ }^{1}$, F. Jauchem ${ }^{2}$, \\ M. Ferriol ${ }^{1}$, B. Benyoucef ${ }^{4}$ and P. Bourson ${ }^{1}$ \\ ${ }^{1}$ Laboratoire Matériaux Optiques, Photoniques et Systèmes, Université Paul Verlaine-Metz, Supelec, UMR \\ CNRS 7132, Département Chimie, IUT de Moselle Est, \\ 12 rue V. Demange, BP 80105, 57503 Saint-Avold Cedex-France \\ ${ }^{2}$ Pôle de Plasturgie de l'Est, Saint Avold, 57500, France \\ ${ }^{3}$ Centre Universitaire Mustapha Stambouli de Mascara, BP : 763, route de Mamounia, 29000, Algérie \\ ${ }^{4}$ Unité de Recherche "Matériaux et Energies Renouvelables » \\ BP : 119, Université de Tlemcen, Faculté des Sciences, 13000, Algérie \\ laarej.merad@metz.supelec.fr
}

\begin{abstract}
Polymerization reactions are based on complex processes that are somewhat difficult to predict via mathematical models, especially without experimental data. A method to investigate the cure of epoxies via in-situ Raman spectroscopy has been developed.

Differential Scanning Calorimetry (DSC) is the industry-standard method for determining the cure of a polymer, but it is a labor-intensive method that is also fairly slow. Raman spectroscopy is used to monitor the cure chemistry of DGEBA (Diglycidyl Ether of Bisphenol A) and to observe in-situ the evolution of the reticulation.
\end{abstract}

Key words : Epoxy resin, process monitoring, DSC, Raman spectroscopy.

\section{INTRODUCTION}

Nowadays, epoxy (EP) resins are intensively used in various technical applications such as coatings, composite matrices, potting compounds, or structural adhesives. Resin Transfer Moulding (RTM) is rapidly gaining acceptance as one of the most promising manufacturing routes for composite structures in applications such as aerospace and automotive industries. However, relatively limited information is available on the chemistry and curing characteristics of thermosetting resins used in such applications. The curing conditions are of crucial importance. Various parameters can influence rheological properties such as composition and amount of hardener and diluents, curing temperature, holding time,... Moreover, addition of insertion elements may influence the polymerization process. In this view, Raman Spectroscopy can be used to monitor locally the composition of materials in relation to the polymerization process. This technique can be used for in-situ control or postmortem analysis of composite materials [1].

The present paper describes the in-situ cure monitoring of a particular epoxy based resin: DGEBA Diglycidyl Ether of Bisphenol A. It is used as high-grade synthetic resins, in the electronical and aeronautical industries. A lot of experimental techniques are suitable for following the rates and extents of cure reactions in thermosets. Among them Differential Scanning Calorimetry (DSC) may be considered as one of the most interesting techniques for macrokinetics analysis of cure reactions of thermosetting systems but it is a destructive method. Our aim is to replace the use of DSC by a non destructive one : Raman spectroscopy [2-4]. 


\section{EXPERIMENTAL}

Reagents: The materials used in this work were a commercially available grade of the Diglycidyl Ether of Bisphenol A ((4-(2, 3 epoxypropoxy) phenyl) propane) abbreviated as DGEBA. It was used under its commercial presentation (Dow Chemical Company; DER 332). The cure agent was 4,4'-DiaminoDiphenylSulfone (DDS). The chemical structures and cure reaction of DGEBA by DDS are given in Figure 1.

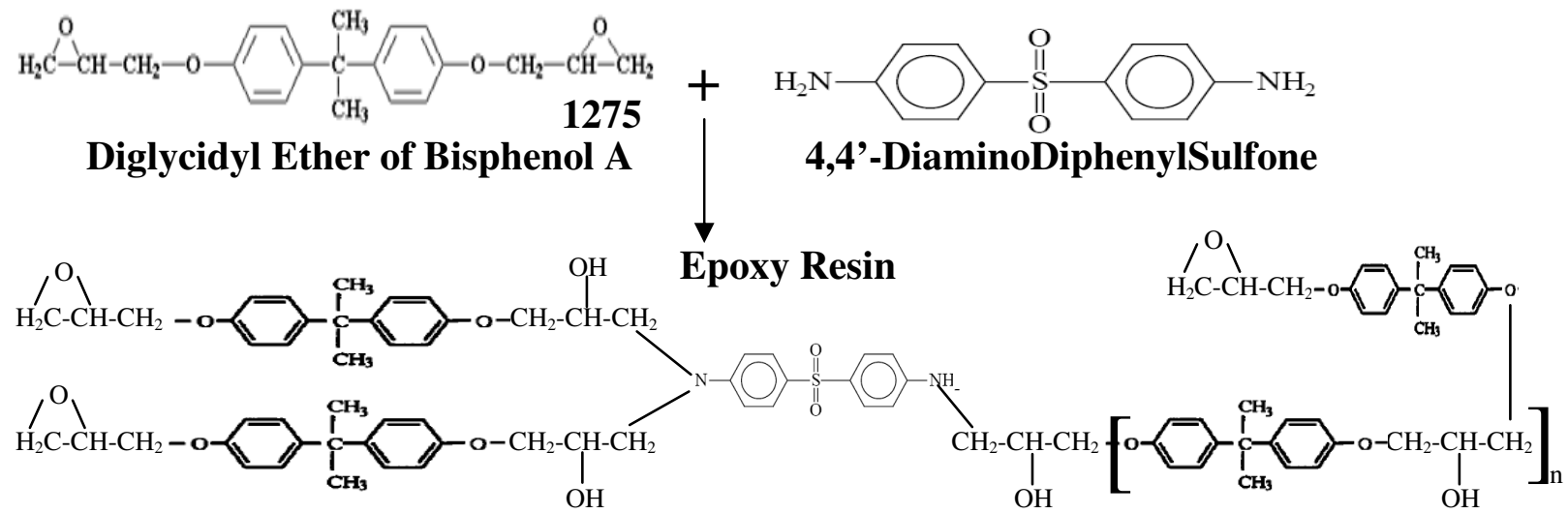

Fig. 1 : Chemical structures and cure reaction of DGEBA by DDS [5]

\section{Apparatus}

DSC: Dynamic scans were performed using a heat-flux DSC (Mettler Toledo TC11) under nitrogen purge. The instrument was calibrated with indium.

Raman: The Raman scattering spectra were recorded with a Jobin Yvon LabRam microRaman system in a backscattering geometry. The analyses were carried out with a laser source at $785 \mathrm{~nm}$. By using a $100 \mathrm{X}$ objective lens, the illuminated spot on the sample surface was focused to about a diameter of $2 \mu \mathrm{m}$. The resolution of the Raman spectra was better than $1 \mathrm{~cm}^{-1}$. Spectra were acquired with a slit width of $50 \mu \mathrm{m}$.

\section{Methods}

DSC: Samples of the mixture of epoxy resin and curing agent were introduced in a furnace maintained at the temperature chosen for the experiment $\left(100,135\right.$ or $\left.150{ }^{\circ} \mathrm{C}\right)$. They were successively removed after different times of heating were elapsed (typically between 1 and 6 hours with a step of 1 hour) and submitted to a DSC analysis with a heating rate of $10^{\circ} \mathrm{C} . \mathrm{min}^{-}$ ${ }^{1}$ across the temperature range from 30 to $350{ }^{\circ} \mathrm{C}$. These scans allowed determining the heat of reaction $\Delta \mathrm{H}_{\text {residual }}$ generated by the curing of the remaining epoxy resin not polymerized in the furnace.

A scan performed directly on a sample of the mixture epoxy resin/curing agent with no heat treatment allowed to determine the heat of reaction $\Delta \mathrm{H}_{\text {total }}$ generated by the complete polymerization process. All these measurements allowed calculating the percentage of reticulation at the different curing times as:

$$
\% \text { reticulation }=100 \cdot \frac{\Delta \mathrm{H}_{\text {total }}-\Delta \mathrm{H}_{\text {residual }}}{\Delta \mathrm{H}_{\text {total }}}
$$

Raman: The mixture DGEBA/DDS was introduced in the furnace accessory of the Raman spectrometer and heated at the desired temperature. Then, the spectra were acquired in-situ every $30 \mathrm{~s}$ to monitor the decrease of the $1275 \mathrm{~cm}^{-1}$ peak corresponding to the epoxide vibration, epoxide functions being consumed by the cure reaction. The $1275 \mathrm{~cm}^{-1}$ peaks were normalized by dividing their intensities by that of $1160 \mathrm{~cm}^{-1}$ peak corresponding to the phenyl 
ring which is unaffected by the curing process and thus remains constant. From these data, the percentage of reticulation was calculated as:

$\%$ reticulation $=100 \cdot \frac{\mathrm{I}_{1275}^{0}-\mathrm{I}_{1275}^{\mathrm{t}}}{\mathrm{I}_{1275}^{0}}$

where $\mathrm{I}_{1275}^{0}$ is the normalized intensity of the $1275 \mathrm{~cm}^{-1}$ peak at room temperature (in the absence of any reaction) and $\mathrm{I}_{1275}^{\mathrm{t}}$ is the normalized intensity of the same peak at different times (typically between 1 and 6 hours with a step of 1 hour).

\section{RESULTS AND DISCUSSION}

The procedure used in the Raman method allows a rather straightforward derivation of quantitative information when one or two monomers are involved. However, it becomes more complex as soon as the reactive medium contains several monomers because the bands of interest from the different monomers overlap [6-7].

Figures 2, 3 and 4 present the Raman spectra obtained at 100,135 and $150^{\circ} \mathrm{C}$ as a function of time. The typical decrease of the $1275 \mathrm{~cm}^{-1}$ peak intensity corresponding to the epoxide group consumption can be observed during all the curing reaction. At $135^{\circ} \mathrm{C}$, almost $70 \%$ of epoxide groups were converted, while during a curing at $150{ }^{\circ} \mathrm{C}, 80 \%$ of epoxide groups were converted. However, it can be expected that the polyesterification reaction will continue also into glassy state but with a significantly decreased rate [8].

Figures 5, 6 and 7 present the comparison of the percentage of reticulation vs time measured by DSC and Raman spectroscopy for the three temperatures investigated. We note a close correlation between the results given by the two methods.
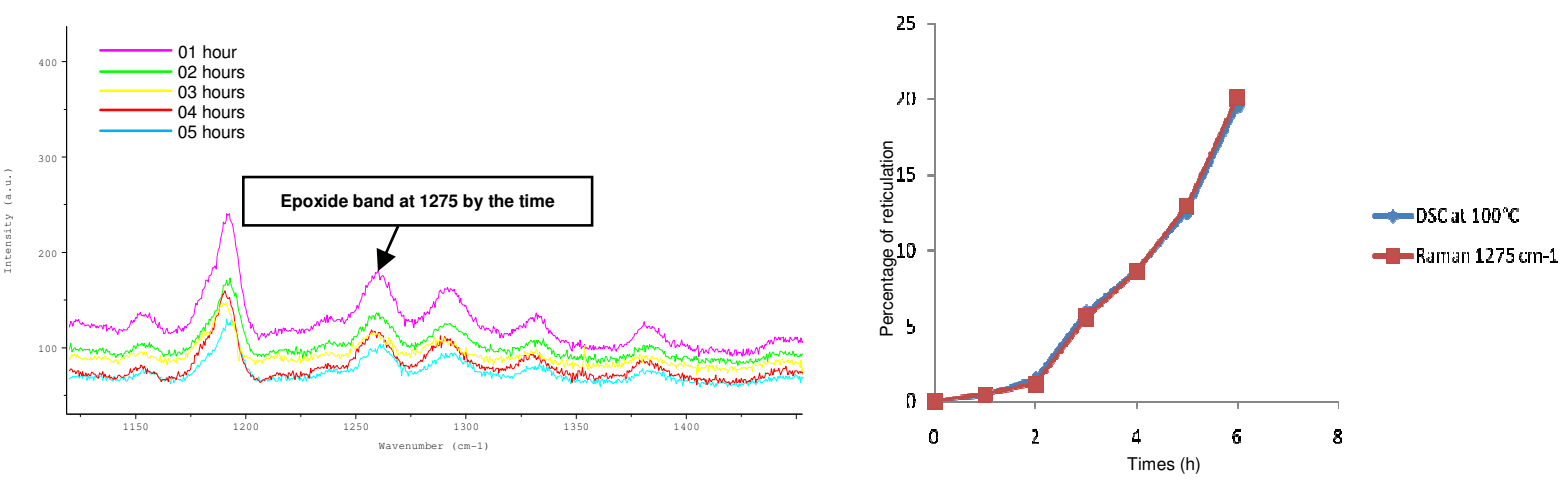

Fig. 2. Raman spectra for RTM6 at $100^{\circ} \mathrm{C}$

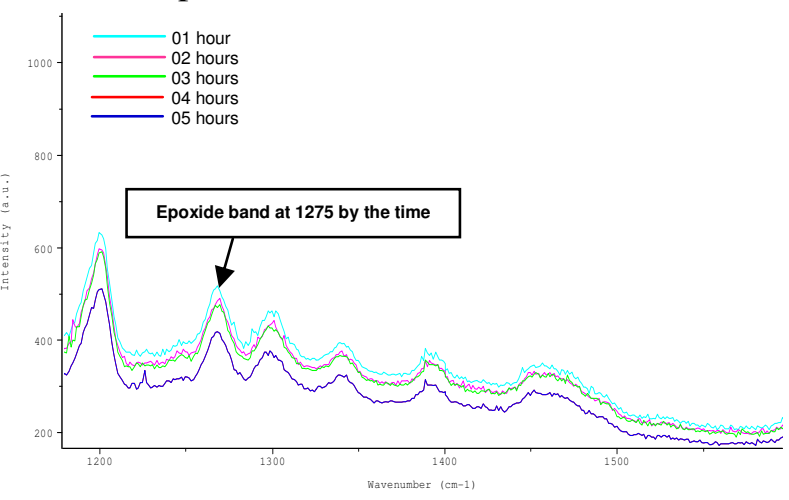

Fig. 3. Raman spectra for RTM6 at $135^{\circ} \mathrm{C}$
Fig. 5. Comparison between the Raman and DSC

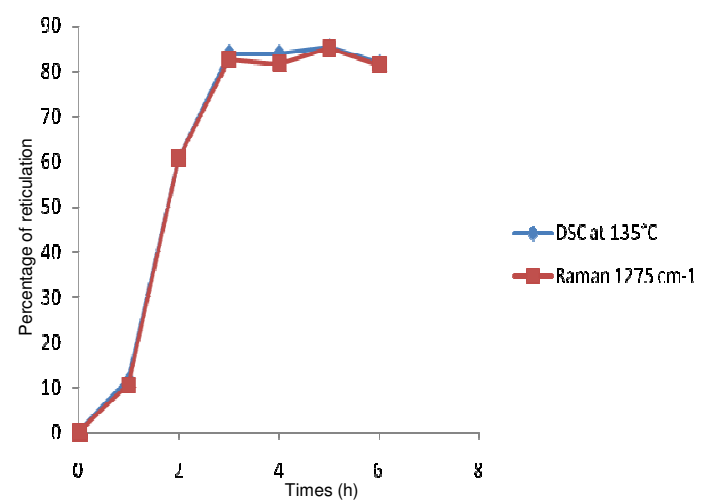

Fig. 6. Comparison between the Raman and DSC 


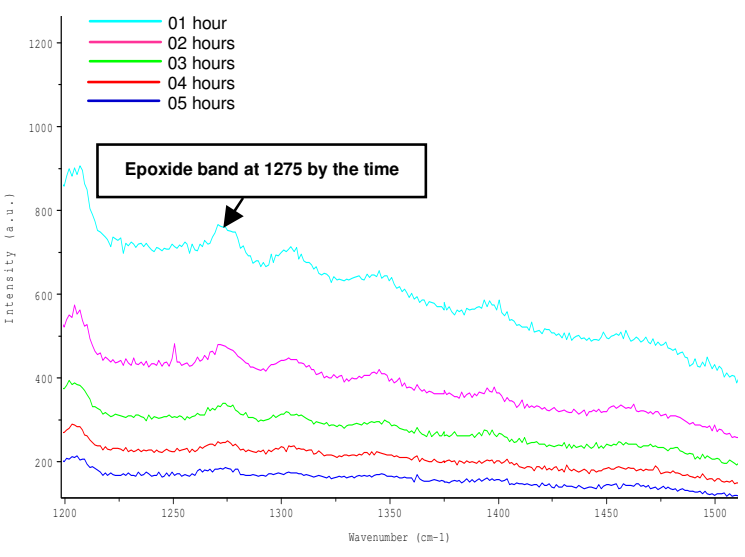

Fig. 4. Raman spectra for RTM6 at $150^{\circ} \mathrm{C}$

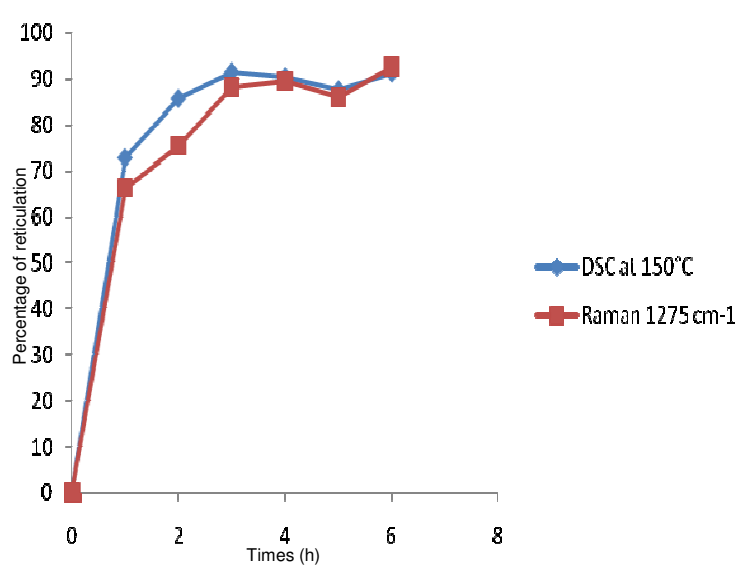

Fig. 7. Comparison between the Raman and DSC

\section{CONCLUSION}

From the comparison established between the conventional DSC investigation method of epoxide cure reactions and the novel Raman spectroscopy method proposed in this work, it can be assessed that Raman spectroscopy is an efficient technique for analysis of epoxy resins because it gives direct information on chemical bonds and has the capability of monitoring insitu and easily curing reactions with access to the control of the kinetics of the reaction.

\section{ACKNOWLEDGEMENTS}

The authors are also grateful to the University Center of Mascara and University of Tlemcen for, their support, encouragement and interest for this work.

\section{REFERENCES}

1. L.S. Penn, H. Wang, Handbook of composites, Edited S.T. Peters, (1998), Published by Chapman \& Hall, London, ISBN 0412540207.

2. P. Navabpour, A. Nesbitt, B. Degamber, G. Ferando, T. Mann, R. Day Comparison of the Curing Kinetics of the RTM6 epoxy Resin System Using Differential Scanning Calorimetry and a MicrowaveHeated Calorimeter, Journal of applied polymer science, vol. 99, (2006), pp. 3658-3668.

3. S. Swier, G.V. Assche, B.V. Mele, Reaction kinetics modeling and Thermal Properties of EpoxyAmines as Measured by Modulated-Temperature DSC. I. Linear Step-Growth Polymerization of DGEBA + Aniline, Journal of applied polymer science, vol. 91, (2004), pp. 2798-2813.

4. P.I. karkanas, I. K. Partridge, Modeling the cure of a commercial Epoxy resin for Application in resin Transfer Moulding, Polymer international, vol. 41, (1996), pp. 183-191.

5. S. Farquharson, W. Smith, M. Shaw, Correlations Between Molecular (Raman) and macroscopic (Rheology) Data for process Monitoring of Thermoset Composite, JPAC $\mathrm{SM}_{\mathrm{SM}}$ process Analytical Chemistry, pp. 45-53.

6. J.F. Aust, K.S. Booksh, C.M. Stellman, R.S. Parnas, M.L. Myrick, Precise Determination of Percent Cure of Epoxide Polymers and Composites via Fiber-Optic Raman Spectroscopy and Multivariate Analysis, Applied spectroscopy, vol. 51, Number 2, (1997), pp. 247-252.

7. R. Montanini, L. d'Acquisto, Simultaneous Measurement of Temperature and Strain in Glass fiberlepoxy Composites by Embedded Fiber Optic Sensors : I. Cure Monitoring, Smart Materials and Structures, vol. 16, (2007), pp. 1718-1726.

8. G.R. Powell, P.A. Crosby, D.N. Waters, C.M. France, R.C. Spooncer, G.F. Fernando, In-situ Cure Monitoring using Optical Fibre Sensor - a Comparative study, Smart Mater Struct., vol. 7, (1998), pp.557-568. 